\title{
Magnetic resonance T1-mapping evaluates the degree of thyroid destruction in patients with autoimmune thyroiditis
}

\author{
Jia Liu', Min Liư', Zhe Chen', Yumei Jia' ${ }^{1}$ and Guang Wang' \\ 'Department of Endocrinology, Beijing Chao-Yang Hospital, Capital Medical University, Beijing, China \\ ${ }^{2}$ Department of Radiology, China-Japan Friendship Hospital, Beijing, China
}

Correspondence should be addressed to G Wang: drwg6688@126.com

\begin{abstract}
Objective: Autoimmune thyroiditis (AIT) is the most common autoimmune thyroid disease. Longitudinal relaxation time mapping (T1-mapping) measured by MRI is a new technique for assessing interstitial fibrosis of some organs, such as heart and liver. This study aimed to evaluate the relationship between T1-mapping value and thyroid function and determine the usefulness of T1-mapping in identifying thyroid destruction in AIT patients.

Methods: This case-control study recruited 57 drug-naïve AIT patients and 17 healthy controls. All participants were given thyroid MRI, and T1-mapping values were measured using a modified look-locker inversion-recovery sequence.

Results: AIT patients had significantly higher thyroid T1-mapping values than the healthy controls $(1.077 \pm 177$ vs $778 \pm 82.9 \mathrm{~ms} ; P<0.01)$. A significant increase in thyroid T1-mapping values was presented along with the increased severity of thyroid dysfunction $(P<0.01)$. Correlation analyses showed that increased thyroid T1-mapping values were associated with higher TSH and lower FT3 and FT4 levels (TSH: $r=0.75$; FT3: $r=-0.47$; FT4: $r=-0.72$; all $P<0.01$ ). Receiver-operating characteristic curve analysis revealed a high diagnostic value of T1-mapping values for the degree of thyroid destruction (area under the curve was $0.95,95 \% \mathrm{Cl}: 0.90-0.99, P<0.01$ ).

Conclusions: AIT patients have higher thyroid T1-mapping values than the healthy controls, and the T1-mapping values increased with the progression of thyroid dysfunction. Thyroid T1-mapping value might be a new index to quantitatively evaluate the degree of thyroid destruction in AIT patients.
\end{abstract}

\author{
Key Words \\ - autoimmune thyroiditis \\ - T1-mapping \\ - thyroid destruction \\ thyroid dysfunction
}

Endocrine Connections (2018) 7, 1315-1321

\section{Introduction}

Autoimmune thyroiditis (AIT) is the most common autoimmune thyroid disease $(1,2)$. Because of varying degree of thyroid destruction, AIT patients manifest with different thyroid function including euthyroidism, subclinical or overt hypothyroidism $(1,2)$. Ultrasound examination is a major imaging detection technique for thyroid diseases and often shows a diffuse hypoechoic or heterogeneous thyroid in AIT patients. However, it is difficult for ultrasound examination to quantitatively evaluate the degree of thyroid destruction (3).

Recently, MRI has been increasingly used for identifying thyroid diseases $(4,5,6,7,8,9)$. Longitudinal relaxation time mapping (T1-mapping) measured by MRI is a new technique for assessing fibrosis of some organs, such as the heart and liver $(10,11)$. Typical thyroid lesions of AIT patients include diffuse lymphocytic infiltration
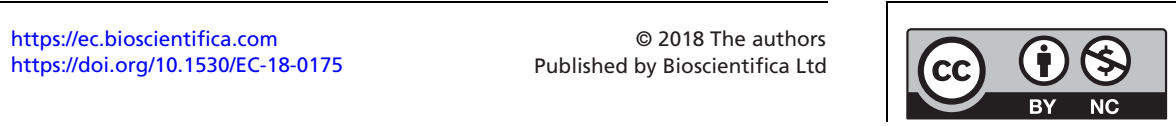

This work is licensed under a Creative Commons Attribution-NonCommercial 4.0 International License. 
with germinal center formation, fibrosis and apoptosis of thyroid epithelial cells (12). The levels of stromal fibrosis mainly reflect the degree of thyroid destruction (12). Many studies, including our previous study, have shown that T1-mapping values can be used to evaluate the fibrosis degree of heart in patients with hypothyroidism, and increased T1-mapping values were significantly correlated with the degree of fibrosis in histology examination $(10,13,14)$. This study aimed to evaluate the relationship between T1-mapping value and thyroid function and determine the usefulness of T1-mapping in identifying thyroid destruction in AIT patients.

\section{Subjects and methods}

Subjects

This study is a case-control study. A total of 57 drug-naïve patients with AIT were recruited from the Endocrinology Department of Beijing Chao-Yang Hospital, Capital Medical University from March 2014 to January 2015. Meanwhile, 17 healthy volunteers without AIT were enrolled as the control group. Free T3 (FT3), free T4 (FT4), thyroid-stimulating hormone (TSH), anti-peroxidase antibody (TPOAb), antithyroglobulin antibody (TgAb) and thyroid ultrasound were performed in all participants. The healthy control subjects were defined based on the following criteria: serum levels of FT3, FT4 and TSH were in the normal ranges (FT3: $2.63-5.71 \mathrm{pmol} / \mathrm{L}$; FT4: 9.10-19.2 pmol/L; TSH: $0.35-4.94 \mathrm{mIU} / \mathrm{L})$; both TPOAb and $\mathrm{TgAb}$ were negative and thyroid ultrasound was normal. AIT was diagnosed by elevated TPOAb (reference range: $0.00-60.0 \mathrm{IU} / \mathrm{mL}$ ) and/or $\mathrm{TgAb}$ (reference range: 0.00-60.0 IU $/ \mathrm{mL}$ ) and typical hypoechoic or heterogeneous thyroid in a thyroid ultrasound (12). Individuals with systemic inflammatory disease, cancer, claustrophobia and metal implants were excluded. No subject had been administered levothyroxine or antithyroid drugs. Individuals who were pregnant, possibly pregnant or ingesting agents known to influence thyroid function were also excluded. All enrolled subjects provided written informed consent, and the Ethics Committee of the Beijing Chao-Yang Hospital, Capital Medical University approved this study's protocol.

\section{Clinical and biochemical measurements}

A standard questionnaire was used to collect information about each patient's health status and medications.
Height and weight were measured to the nearest $0.1 \mathrm{~cm}$ and $0.1 \mathrm{~kg}$ by the same trained group, respectively. Venous blood samples were obtained after overnight fasting. All participants' plasma samples were stored at $-80^{\circ} \mathrm{C}$. FT3, FT4 and TSH were measured by electrochemiluminescence immunoassay using an Abbott Architect i2000 (Abbott Diagnostics, Abbott Park). The serum concentrations of $\mathrm{TgAb}$ and TPOAb were detected by chemiluminescent immunoassay. High-sensitivity C-reactive protein (hsCRP) was measured with an immunonephelometric assay. BMI was calculated as the weight in kilograms divided by the height in meters squared. A well-trained ultrasound physician assessed the thyroid ultrasound.

\section{Thyroid magnetic resonance imaging}

Thyroid MRI was performed with participants in a supine position using 3 Tesla scanners on a Tim Trio System (Siemens Healthcare, Erlangen, Germany). Cine images were acquired by gapless whole thyroid coverage, and slice thickness for cine was $8 \mathrm{~mm}$. All routine MRI and maps were analyzed with Argus (SYNGO MMWP Workstation, Siemens AG). T1-mapping is a parametric reconstructed image analyzed using a modified looklocker inversion-recovery (MOLLI) sequence without contrast administration $(14,15)$. Circle region of interests (ROIs) were drawn to thyroid tissue in the AIT patients and healthy subjects. Shimming and center frequency adjustments were performed to generate off-resonance artifact-free images. Two expert radiologists (M L, 11 years of experience; F F Y, 4 years of experience) who were blinded to the groups performed image analyses for T1-mapping value. The T1-mapping values of left (T1-L-Thyroid) and right (T1-R-Thyroid) lobar thyroid were calculated by an average across all of the available slices. The final result of thyroid T1-mapping value (T1-Thyroid) was determined by averaging the values of T1-L-Thyroid and T1-R-Thyroid.

\section{Statistical analysis}

Data were analyzed using SPSS 21.0 (SPSS) and MedCalc 15.10 (MedCalc Software, Mariakerke, Belgium). Normally distributed variables were expressed as mean \pm standard deviation (S.D.), while variables with a skewed distribution including TSH, TPOAb, TgAb and hsCRP were given as the median and upper and lower quartiles. The skew distributional variables were log-transformed before analysis. The differences between the control and AIT groups were analyzed by independent Student's $t$-test

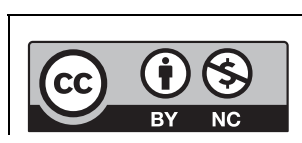

This work is licensed under a Creative Commons Attribution-NonCommercial 4.0 International License. 
or the Mann-Whitney $U$ test using SPSS 21.0. And the differences among the control and three AIT subgroups were analyzed by ANOVA test using SPSS 21.0. The proportions were analyzed using chi-square tests using SPSS 21.0. Spearman or Pearson analysis was used to evaluate the correlations using SPSS 21.0. A receiveroperating characteristic (ROC) curve was used to evaluate the diagnostic performance of thyroid T1-mapping value for the degree of thyroid destruction, and Youden index was used as a criterion for selecting the optimum cutoff point using MedCalc 15.10. The results were considered statistically significant with two-tailed analyses, $P<0.05$.

\section{Results}

\section{Baseline characteristics of the control and AIT groups}

The baseline characteristics of the control and AIT groups are summarized in Table 1. No significant difference in age and gender was observed between the two groups. Body weight and BMI were higher in the AIT patients than in the controls (body weight: $66.4 \pm 12.5$ vs $59.4 \pm 10.5 \mathrm{~kg}$; BMI: $25.1 \pm 4.5$ vs $22.0 \pm 3.1 \mathrm{~kg} / \mathrm{m}^{2}$; all $P<0.05$; Table 1 ). AIT patients had significantly lower FT3 and FT4 levels and higher TSH, TPOAb and TgAb levels than the controls (FT3: $3.17 \pm 1.15$ vs $4.33 \pm 0.64 \mathrm{pmol} / \mathrm{L}$; FT4: $8.49 \pm 3.73$ vs $14.2 \pm 1.67 \mathrm{pmol} / \mathrm{L}$; TSH: $61.6(6.09-100)$ vs 1.89 (1.42-3.08) mIU/L; TPOAb: 1141 (247-2000) vs $0.4 \quad(0.2-0.6) \mathrm{IU} / \mathrm{mL} ; \quad \mathrm{TgAb}: 141 \quad$ (37.9-849) vs $1.3(0.8-35.9) \mathrm{IU} / \mathrm{mL}$; all $P<0.01$; Table 1$)$. We also found higher levels of hsCRP in the AIT patients than in the control group (0.47 (0.00-1.52) vs $0.00(0.00-0.04) \mathrm{mg} / \mathrm{L}$, $P<0.01$; Table 1). Native MOLLI showed significantly higher thyroid T1-mapping values in AIT patients than in healthy controls $(1077 \pm 177$ vs $778 \pm 82.9$ ms; $P<0.01$; Table 1).

\section{Clinical characteristics in AIT patients with different thyroid function}

AIT patients were further divided into three subgroups: euthyroid AIT group (EU group, $n=12$ ), subclinicalhypothyroid group (SHO group, $n=16$ ) and overthypothyroid group (HO group, $n=29$ ). The EU group was defined as AIT patients with normal levels of FT4 and TSH. The SHO group was diagnosed as AIT patients with increased serum TSH levels and normal FT4 levels, and the HO group was diagnosed as AIT patients with increased serum TSH levels and decreased FT4 levels.

The baseline characteristics of the control group and the three AIT subgroups are shown in Table 1. A significant trend of FT3, FT4, TSH and TPOAb was presented among the control group, EU group, SHO group and HO groups (all $P<0.01$; Table 1). We also found a significant increase in thyroid T1-mapping value along with the increased

Table 1 The baseline characteristics of the control and AIT groups.

\begin{tabular}{|c|c|c|c|c|c|c|c|}
\hline \multirow[b]{2}{*}{ Parameters } & \multirow{2}{*}{$\begin{array}{c}\text { Control group } \\
(n=17)\end{array}$} & \multicolumn{4}{|c|}{ AIT group } & \multirow[b]{2}{*}{$P_{1}$} & \multirow[b]{2}{*}{$\boldsymbol{P}_{2}$} \\
\hline & & $\operatorname{ALL}(n=57)$ & EU group $(n=12)$ & SHO group $(n=16)$ & HO group $(n=29)$ & & \\
\hline Age (years) & $34.8 \pm 9.1$ & $40.4 \pm 12.8$ & $49.3 \pm 13.7$ & $40.6 \pm 15.1$ & $36.7 \pm 9.3$ & 0.10 & 0.06 \\
\hline $\begin{array}{l}\text { Gender, } \\
\text { males/females }(n)\end{array}$ & $2 / 15$ & $6 / 51$ & $2 / 10$ & $1 / 15$ & $3 / 26$ & 0.59 & 0.87 \\
\hline Body weight (kg) & $59.4 \pm 10.5$ & $66.4 \pm 12.5$ & $64.2 \pm 11.5$ & $64.0 \pm 15.6$ & $68.4 \pm 11.3$ & 0.04 & 0.12 \\
\hline BMI $\left(\mathrm{kg} / \mathrm{m}^{2}\right)$ & $22.0 \pm 3.1$ & $25.1 \pm 4.5$ & $24.5 \pm 4.8$ & $23.7 \pm 5.2$ & $26.0 \pm 3.9$ & 0.01 & 0.02 \\
\hline FT3 (pmol/L) & $4.33 \pm 0.64$ & $3.17 \pm 1.15$ & $3.88 \pm 1.29$ & $3.97 \pm 0.71$ & $2.45 \pm 0.78$ & $<0.01$ & $<0.01$ \\
\hline FT4 (pmol/L) & $14.2 \pm 1.67$ & $8.49 \pm 3.73$ & $12.6 \pm 4.12$ & $10.6 \pm 2.06$ & $5.79 \pm 1.54$ & $<0.01$ & $<0.01$ \\
\hline TSH (mIU/L) & $1.89(1.42-3.08)$ & $61.6(6.09-100)$ & $2.59(1.39-4.26)$ & $16.4(6.54-32.6)$ & $100(93.1-100)$ & $<0.01$ & $<0.01$ \\
\hline TPOAb (IU/mL) & $0.4(0.2-0.6)$ & $1141(247-2000)$ & 994 (529-2000) & $1261(303-2000)$ & $1287(12.6-2000)$ & $<0.01$ & $<0.01$ \\
\hline TgAb (IU/mL) & $1.3(0.8-35.9)$ & 141 (37.9-849) & $500(50.8-909)$ & $159(28.1-620)$ & 104 (34.2-1597) & $<0.01$ & 0.14 \\
\hline hsCRP (mg/L) & $0.00(0.00-0.04)$ & $0.47(0.00-1.52)$ & $2.47(0.20-4.39)$ & $0.12(0.00-1.46)$ & $0.60(0.07-1.48)$ & $<0.01$ & 0.07 \\
\hline T1-L-Thyroid (ms) & $776 \pm 84.5$ & $1078 \pm 179$ & $901 \pm 91.0$ & $1048 \pm 147$ & $1171 \pm 163$ & $<0.01$ & $<0.01$ \\
\hline T1-R-Thyroid (ms) & $780 \pm 83.0$ & $1072 \pm 177$ & $904 \pm 88.9$ & $1051 \pm 141$ & $1160 \pm 170$ & $<0.01$ & $<0.01$ \\
\hline T1-Thyroid (ms) & $778 \pm 82.9$ & $1077 \pm 177$ & $902 \pm 88.5$ & $1050 \pm 142$ & $1167 \pm 163$ & $<0.01$ & $<0.01$ \\
\hline
\end{tabular}

Data are means \pm S.D. unless indicated otherwise. TSH, TPOAb, TgAb and hsCRP are shown as median, upper and lower quartiles. AIT patients were further divided into three subgroups: euthyroid AIT group (EU group), subclinical-hypothyroid group (SHO group) and overt-hypothyroid group (HO group). $P_{1}$ : the AIT group vs the control group, analyzed by independent Student's $t$-test or the Mann-Whitney $U$ test using SPSS 21.0 . $P_{2}$ : the trend among the control group, the EU group, the SHO group and the HO group, analyzed by ANOVA test using SPSS 21.0 .

BMI, body mass index; FT3, free T3; FT4, free T4; hsCRP, high-sensitivity C-reactive protein; T1-Thyroid, the averages of T1-mapping values of the left and right lobar thyroids; T1-L-Thyroid, the T1-mapping values of the left lobar thyroid; T1-R-Thyroid, the T1-mapping values of the right lobar thyroid; TgAb, antithyroglobulin antibodies; TPOAb, antithyroid peroxidase antibodies.

https://ec.bioscientifica.com

https://doi.org/10.1530/EC-18-0175 (c) 2018 The authors Published by Bioscientifica Ltd

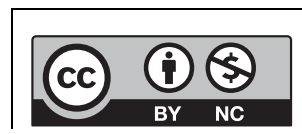

This work is licensed under a Creative Commons Attribution-NonCommercial 4.0 International License. 

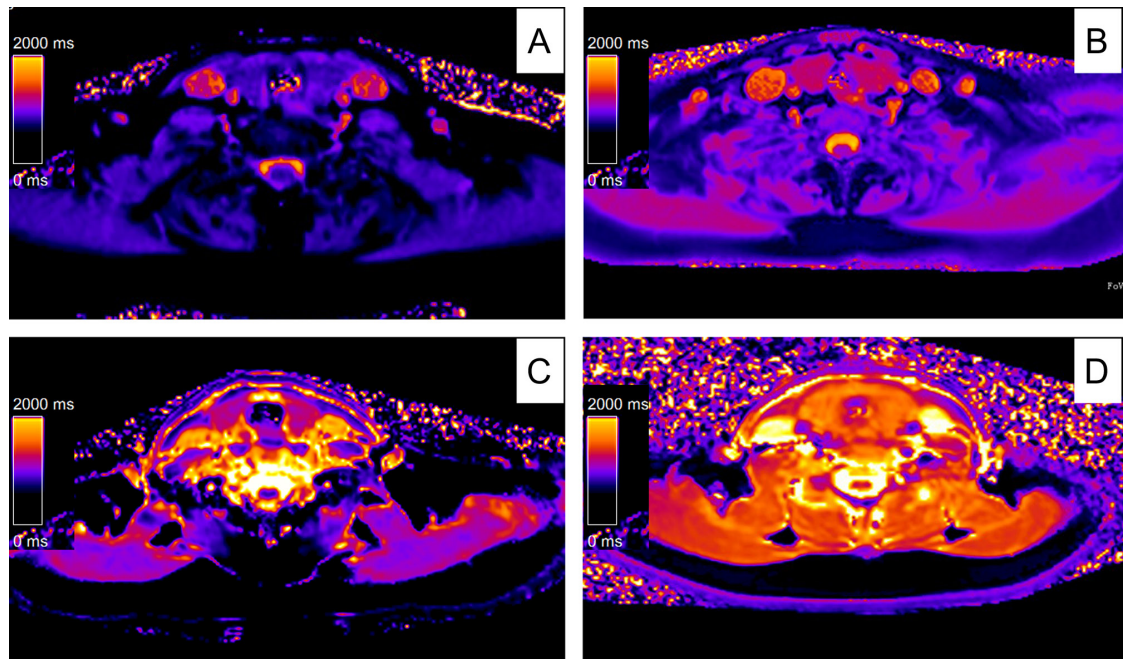

\section{Figure 1}

Color maps of thyroid T1-mapping based on a modified look-locker inversion-recovery (MOLLI) sequence. (A) A healthy control subject ( $\mathrm{T} 1=837 \mathrm{~ms})$, (B) a patient from EU group ( $\mathrm{T} 1=1005 \mathrm{~ms})$, (C) a patient from SHO group $(\mathrm{T} 1=1257 \mathrm{~ms})$ and (D) a patient from HO group $(\mathrm{T} 1=1430 \mathrm{~ms})$. EU group, euthyroid AIT group; HO group, overt-hypothyroid AIT group; SHO group, subclinical-hypothyroid AIT group.

severity of thyroid dysfunction (among the control group, the EU group, the SHO group and the HO group) $(P<0.01$; Figs 1, 2 and Table 1).

\section{Correlation between thyroid T1-mapping values and thyroid function}

We next assessed whether thyroid T1-mapping values were related to thyroid function. Remarkably, thyroid T1-mapping values were significantly associated with the serum levels of FT3 and FT4 (FT3: $r=-0.47,95 \%$ CI: -0.63 to $-0.27, P<0.01$, Fig. 3A; FT4: $r=-0.72,95 \%$ CI: -0.81 to $-0.59, P<0.01$, Fig. $3 \mathrm{~B})$. We also observed a significant positive correlation between thyroid T1-mapping values and TSH ( $r=0.75,95 \%$ CI: 0.62-0.84, $P<0.01$, Fig. 3C). In addition, thyroid T1-mapping values were also associated with the levels of TPOAb, TgAb and hsCRP (TPOAb: $r=0.51,95 \%$ CI: 0.31-0.67, $P<0.01$; TgAb: $r=0.45,95 \%$ CI: 0.24-0.63, $P<0.01$; hsCRP: $r=0.38,95 \%$ CI: $0.10-0.60$, $P<0.01)$.

\section{Diagnostic performance of thyroid T1-mapping values for the degrees of thyroid destruction}

ROC curve analysis revealed a very high diagnostic value of thyroid T1-mapping values for the degrees of thyroid destruction. If we defined thyroid destruction as the thyroid function was subclinical hypothyroidism and overt hypothyroidism, the area under the curve (AUC) was 0.95 (95\% CI: 0.90-0.99, $P<0.01$, Fig. 4A). The optimal cutoff point estimated from Youden index was 963.2, with a sensitivity of $88.6 \%$ and a specificity of $90.0 \%$. If we defined overt hypothyroidism as thyroid destruction, the AUC was 0.88 (95\% CI: 0.79-0.96, P<0.01, Fig. 4B).
The optimal cutoff point estimated from Youden index was 1027.3 , with a sensitivity of $75.0 \%$ and a specificity of $88.9 \%$.

\section{Discussion}

In the present study, thyroid T1-mapping values were significantly higher in the AIT patients than the healthy controls. A significant increase in thyroid T1-mapping values was presented along with the increased severity of thyroid dysfunction. Increased thyroid T1-mapping values were associated with higher TSH and lower FT3 and FT4 levels. In addition, significant positive correlations between thyroid T1-mapping values and the levels of

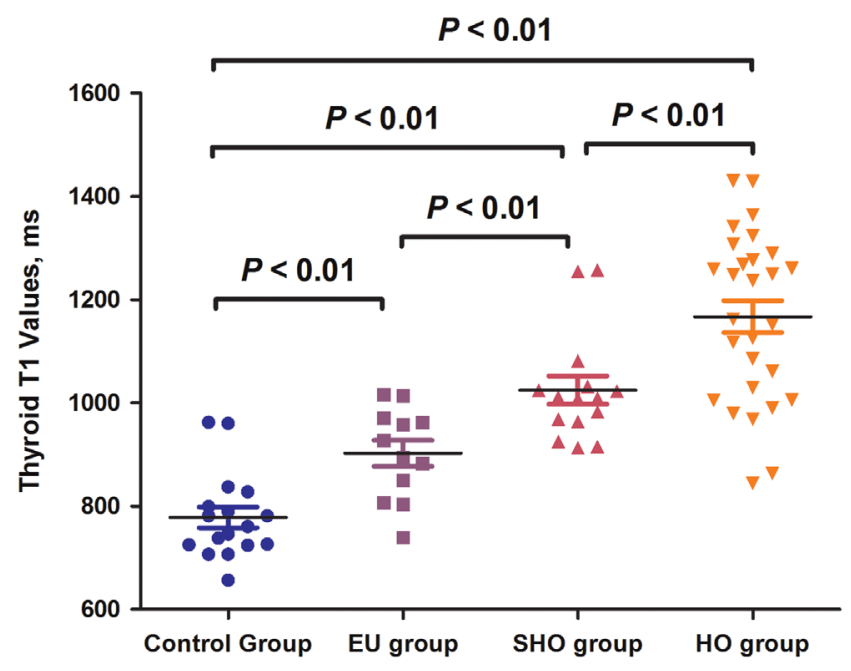

Figure 2

The thyroid T1-mapping values of the control, EU, SHO and $\mathrm{HO}$ groups. EU group, euthyroid AIT group; HO group, overt-hypothyroid AIT group; SHO group, subclinical-hypothyroid AIT group. https://ec.bioscientifica.com

https://doi.org/10.1530/EC-18-0175 (c) 2018 The authors Published by Bioscientifica Ltd

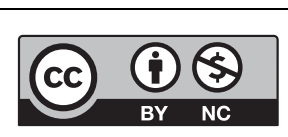

This work is licensed under a Creative Commons Attribution-NonCommercial 4.0 International License. 
A

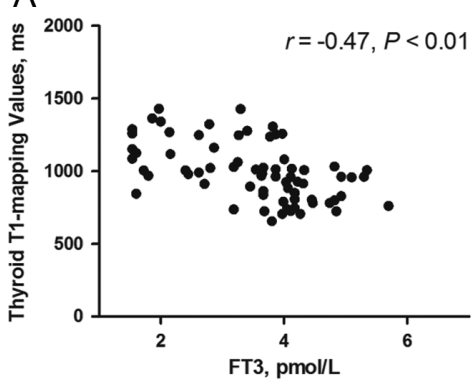

B

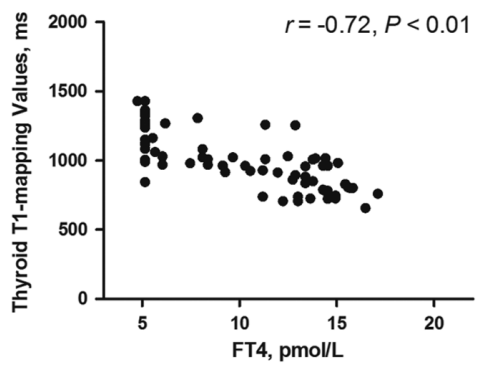

C

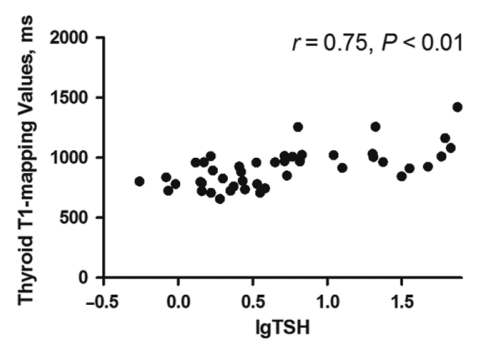

Figure 3

The correlation between thyroid T1-mapping values and the levels of FT3 (A), FT4 (B) and TSH (C). Thyroid T1-mapping values were significantly associated with the serum levels of FT3, FT4 and TSH (FT3: $r=-0.47,95 \% \mathrm{Cl}:-0.63$ to $-0.27, P<0.01, \mathrm{~A} ; \mathrm{FT} 4: r=-0.72,95 \% \mathrm{Cl}:-0.81$ to $-0.59, P<0.01, \mathrm{~B}$; TSH: $r=0.75,95 \% \mathrm{Cl}: 0.62-0.84, P<0.01, \mathrm{C})$.

hsCRP, TPOAb and TgAb were also observed. ROC curve analysis revealed a very high diagnostic value of thyroid T1-mapping values for the degree of thyroid destruction.

Magnetic resonance T1-mapping is a parametric reconstructed image analyzed using a MOLLI sequence and has been considered as an emerging technique for evaluating tissue fibrosis $(10,11,14)$. Many previous studies have shown that increased T1-mapping values were correlated well with the degree of histological fibrosis (13). In addition, T1-mapping value was much more sensitive to water and better reflected myocardial edema than conventional T2-weighted value $(16,17)$. AIT is characterized by lymphocytic infiltration in the thyroid gland (1). T lymphocytes infiltrated in the thyroid induce edema and apoptosis of thyroid follicular cells by cell-mediated immunity $(12,18)$. The presence of diffuse lymphocytic infiltration, fibrosis, edema and apoptosis of thyroid epithelial cells is the typical lesions for thyroid T1-mapping values were significantly higher in the AIT patients, and a significant increase in thyroid T1-mapping values was presented along with the increased severity of thyroid dysfunction. ROC curve analysis demonstrated a very high diagnostic value of thyroid T1-mapping values for the degree of thyroid destruction. Therefore, thyroid T1-mapping values might be a potential index, which was related to the degree of thyroid destruction for AIT patients.

The development of AIT is associated with both cellular and humoral immunity (2). In addition to inducing apoptosis of thyroid follicular cells, T lymphocytes infiltrated in the thyroid produce large amounts of inflammatory cytokines and cause a chronic inflammatory status (18). Increased inflammatory cytokines further enhance the inflammatory response of $\mathrm{T}$ and $\mathrm{B}$ lymphocytes and induce the apoptosis of thyroid follicular cells and the production of thyroid autoantibodies (18). TPOAb and $\mathrm{TgAb}$ are the two important antibodies of humoral immunity, and mainly produced by lymphocytes infiltrated in thyroid $(19,20)$. $\mathrm{TPOAb}$ induces cellular cytotoxicity and complement system and further deteriorates the inflammatory status and thyroid destruction $(18,19,20)$. In the present study, AIT patients have significantly increased levels of hsCRP, TPOAb and TgAb, and their thyroid T1-mapping values
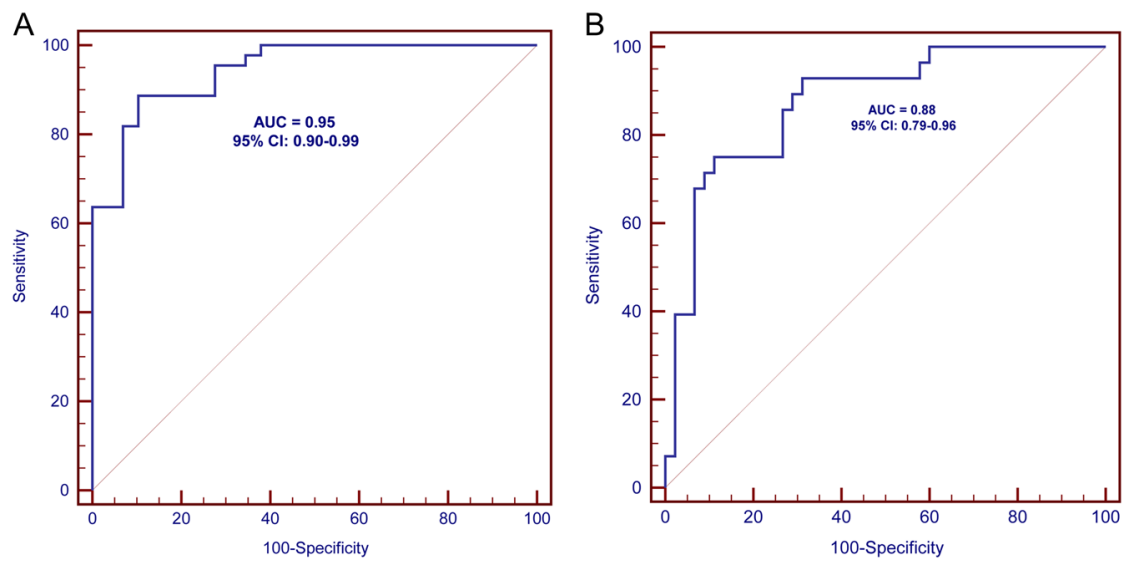
Figure 4
Receiver-operating characteristic (ROC) curves of thyroid T1-mapping values for the degrees of thyroid destruction. If we defined severe thyroid destruction when the thyroid function was subclinical hypothyroidism and overt hypothyroidism, the area under the curve (AUC) was 0.95 ( $95 \%$ Cl: $0.90-0.99, P<0.01$, A). If we defined overt hypothyroidism as severe thyroid destruction, the AUC was 0.88 (95\% Cl: 0.79-0.96, $P<0.01, \mathrm{~B})$.

https://ec.bioscientifica.com https://doi.org/10.1530/EC-18-0175 (c) 2018 The authors Published by Bioscientifica Ltd

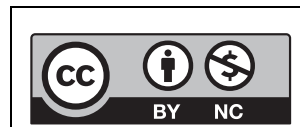

This work is licensed under a Creative Commons Attribution-NonCommercial 4.0 International License. 
were significantly associated with the levels of hsCRP, $\mathrm{TPOAb}$ and TgAb. These results further supported that thyroid T1-mapping values were related to the degree of thyroid destruction.

In general, the state of an organ is identified by evaluating its structure and function. Blood test is an important diagnostic tool for assessing thyroid function; however, there is a lack of examinations for assessing the change of thyroid structure, especially the degree of thyroid destruction in AIT patients. As a major imaging detection technique for thyroid diseases, ultrasound examination often shows a diffuse hypoechoic or heterogeneous thyroid in AIT patient (3). However, ultrasound examination cannot quantitatively evaluate the degree of thyroid destruction (3). Thyroid MRI is a noninvasive examination technique without the risk of radiation and contrast medium injection $(4,5)$. In recent years, thyroid MRI has been increasingly used in the diagnosis of thyroid diseases $(4,5,6,7,8,9)$. For example, diffusion-weighted MRI was well applied in identifying autoimmune thyroid disease and malignant thyroid nodules $(4,5,6,7,8,9)$. T1-mapping is a new parametric reconstructed image of MRI. Thyroid T1-mapping values increased with the progression of thyroid dysfunction in AIT patients. Therefore, Thyroid T1-mapping values could be considered as a potential index to quantitatively evaluate the degree of thyroid destruction for AIT patients. Furthermore, AIT patients, even with normal thyroid function, still had higher thyroid T1-mapping values than the healthy controls. The AIT patients with more severe thyroid destruction are much more likely to develop hypothyroidism, so careful follow-up examination should be required in the AIT patients with higher thyroid T1-mapping values.

The present study has several limitations. First, this study was a case-control study. Second, this study was carried out in a single center with a relatively small sample size. It might have some confounders to influence the results. Therefore, it is difficult for the present study to suggest a generalizable result. Moreover, the cost-benefit ratio is another aspect that should be considered. Nevertheless, this study proposed a new viewpoint for assessing thyroid structure in AIT patients.

In conclusions, AIT patients had significantly higher thyroid T1-mapping values than the healthy controls, and the T1-mapping values increased with the progression of thyroid dysfunction. Thyroid T1-mapping values might be a new index to quantitatively evaluate the degree of thyroid destruction in AIT patients.

\section{Declaration of interest}

The authors declare that there is no conflict of interest that could be perceived as prejudicing the impartiality of the research reported.

\section{Funding}

This work was supported by grants from the Chinese National Natural Science Foundation (No. 81600657) and Capital Funds for Health Improvement and Research (No. 2018-4-2034) to J L and the Chinese National Natural Science Foundation (No. 81770792) and Capital Clinical Research Foundation of Beijing Municipal Commission of Science and Technology (No. Z161100000516069) to G W and Chinese National Natural Science Foundation (No.30900364) to M L.

\section{Author contribution statement}

$\mathrm{J} \mathrm{L}, \mathrm{M} \mathrm{L}$ and $\mathrm{G} \mathrm{W}$ conceived and designed the experiments; J L, M L and Z C performed the experiments and $Y \mathrm{M} \mathrm{J} ; \mathrm{J} L$ and $\mathrm{G}$ W analyzed the data; $\mathrm{J} \mathrm{L}$ wrote the paper.

\section{References}

1 Antonelli A, Ferrari SM, Corrado A, Di Domenicantonio A \& Fallahi P. Autoimmune thyroid disorders. Autoimmunity Reviews 2015 14 174-180. (https://doi.org/10.1016/j.autrev.2014.10.016)

2 Weetman AP. Autoimmune thyroid disease: propagation and progression. European Journal of Endocrinology 2003148 1-9. (https:// doi.org/10.1530/eje.0.1480001)

3 Dobruch-Sobczak K, Jedrzejowski M, Jakubowski W \& Trzebinska A. Errors and mistakes in ultrasound diagnostics of the thyroid gland. Journal of Ultrasonography 201414 61-73. (https://doi.org/10.15557/ JoU.2014.0006)

4 Ozturk T, Bozgeyik Z, Ozturk F, Burakgazi G, Akyol M, Coskun S, Ozkan Y \& Ogur E. The role of diffusion weighted MR imaging for differentiation between Graves' disease and Hashimoto thyroiditis. European Review for Medical and Pharmacological Sciences 201519 2798-2803.

5 Takashima S, Fukuda H, Fujita N, Iwatani Y \& Nakamura H. Hashimoto thyroiditis: correlation of MR imaging signal intensity with histopathologic findings and thyroid function test results. Radiology 1995197 213-219. (https://doi.org/10.1148/ radiology.197.1.7568826)

6 Erdem G, Erdem T, Muammer H, Mutlu DY, Firat AK, Sahin I \& Alkan A. Diffusion-weighted images differentiate benign from malignant thyroid nodules. Journal of Magnetic Resonance Imaging 201031 94-100. (https://doi.org/10.1002/jmri.22000)

7 Shi R, Yao Q, Wu L, Zhou Q, Lu Q, Gao R, Hu J, Kao L, Bains A, Yan Z, et al. T2* Mapping at 3.0T MRI for differentiation of papillary thyroid carcinoma from benign thyroid nodules. Journal of Magnetic Resonance Imaging 201643 956-961. (https://doi.org/10.1002/ jmri.25041)

8 Taviani V, Nagala S, Priest AN, McLean MA, Jani P \& Graves MJ. 3T diffusion-weighted MRI of the thyroid gland with reduced distortion: preliminary results. British Journal of Radiology $2013 \mathbf{8 6} 20130022$. (https://doi.org/10.1259/bjr.20130022)

9 Lu Y, Hatzoglou V, Banerjee S, Stambuk HE, Gonen M, Shankaranarayanan A, Mazaheri Y, Deasy JO, Shaha AR, Tuttle RM, et al. Repeatability investigation of reduced field-of-view diffusionweighted magnetic resonance imaging on thyroid glands. Journal of Computer Assisted Tomography 201539 334-339. (https://doi. org/10.1097/RCT.0000000000000227)

10 Puntmann VO, Voigt T, Chen Z, Mayr M, Karim R, Rhode K, Pastor A, Carr-White G, Razavi R, Schaeffter T, et al. Native T1

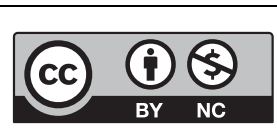

This work is licensed under a Creative Commons Attribution-NonCommercial 4.0 International License. 
mapping in differentiation of normal myocardium from diffuse disease in hypertrophic and dilated cardiomyopathy. JACC: Cardiovascular Imaging 20136 475-484. (https://doi.org/10.1016/j. jcmg.2012.08.019)

11 Ding Y, Rao S, Yang L, Chen C \& Zeng M. Comparison of the effect of region-of-interest methods using gadoxetic acid-enhanced MR imaging with diffusion-weighted imaging on staging hepatic fibrosis. La Radiologia Medica 2016121 821-827. (https://doi.org/10.1007/ s11547-016-0669-7)

12 Melmed S, Polonsky KS, Reed Larsen P \& Kronenberg HM. In Williams Textbook of Endocrinology, 13th ed., pp 441-443. Philadelphia: Elsevier, 2016.

13 Bull S, White SK, Piechnik SK, Flett AS, Ferreira VM, Loudon M, Francis JM, Karamitsos TD, Prendergast BD, Robson MD, et al. Human non-contrast T1 values and correlation with histology in diffuse fibrosis. Heart 201399 932-937. (https://doi.org/10.1136/ heartjnl-2012-303052)

14 Gao X, Liu M, Qu A, Chen Z, Jia Y, Yang N, Feng X, Liu J, Xu Y, Yang $X$, et al. Native magnetic resonance T1-mapping identifies diffuse myocardial injury in hypothyroidism. PLOS ONE 201611 e0151266. (https://doi.org/10.1371/journal.pone.0151266)

15 Messroghli DR, Radjenovic A, Kozerke S, Higgins DM, Sivananthan MU \& Ridgway JP. Modified Look-Locker inversion recovery (MOLLI) for high-resolution T1 mapping of the heart. Magnetic Resonance in Medicine 200452 141-146. (https://doi. org/10.1002/mrm.20110)
16 Ferreira VM, Piechnik SK, Dall'Armellina E, Karamitsos TD, Francis JM, Ntusi N, Holloway C, Choudhury RP, Kardos A, Robson MD, et al. T(1) mapping for the diagnosis of acute myocarditis using CMR: comparison to T2-weighted and late gadolinium enhanced imaging. JACC: Cardiovascular Imaging 20136 1048-1058. (https://doi.org/10.1016/j.jcmg.2013.03.008)

17 Ferreira VM, Piechnik SK, Dall'Armellina E, Karamitsos TD, Francis JM, Choudhury RP, Friedrich MG, Robson MD \& Neubauer S. Non-contrast T1-mapping detects acute myocardial edema with high diagnostic accuracy: a comparison to T2-weighted cardiovascular magnetic resonance. Journal of Cardiovascular Magnetic Resonance 201214 42. (https://doi.org/10.1186/1532-429X-14-42)

18 Mikos H, Mikos M, Obara-Moszynska M \& Niedziela M. The role of the immune system and cytokines involved in the pathogenesis of autoimmune thyroid disease (AITD). Endokrynologia Polska 201465 150-155. (https://doi.org/10.5603/EP.2014.0021)

19 Czarnocka B, Janota-Bzowski M, McIntosh RS, Asghar MS, Watson PF, Kemp EH, Carayon P \& Weetman AP. Immunoglobulin G kappa antithyroid peroxidase antibodies in Hashimoto's thyroiditis: epitope-mapping analysis. Journal of Clinical Endocrinology and Metabolism 199782 2639-2644. (https://doi.org/10.1210/ jcem.82.8.4124)

20 Prummel MF \& Wiersinga WM. Thyroidperoxidase autoantibodies in euthyroid subjects. Best Practice and Research in Clinical Endocrinology and Metabolism 200519 1-15. (https://doi.org/10.1016/j. beem.2004.11.003)

Received in final form 13 July 2018

Accepted 13 August 2018

Accepted Preprint published online 17 August 2018 https://ec.bioscientifica.com https://doi.org/10.1530/EC-18-0175 (c) 2018 The authors Published by Bioscientifica Ltd

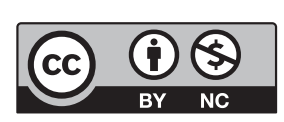

This work is licensed under a Creative Commons Attribution-NonCommercial 4.0 International License. 